\title{
Síndrome Atra: experiência de 10 anos
}

\author{
ATRA Syndrome: 10 year experience
}

\author{
Ronald Feitosa Pinheiro, ${ }^{1}$ Luis Arthur Flores Pelloso, ${ }^{2}$ Mihoko Yamamoto, ${ }^{3}$ \\ Maria de Lourdes Lopes Ferrari Chauffaille e e osé Orlando Bordim ${ }^{4}$
}

\begin{abstract}
Resumo
A leucemia promielocítica aguda (LPA) é um subtipo de leucemia mielóide aguda (LM A) responsável por 10\% de todas as LM As. G eralmente, o tratamento da LPA consiste de quimioterapia e uso de ácido transretinóico (AT RA). 0 maior efeito colateral do ATRA é a "Síndrome ATRA", que ocorre com uma freqüência de quase $30 \%$. Estudamos a apresentação clínica e a incidência da Síndrome AT RA em pacientes com LPA admitidos no H ospital São Paulo (da Escola Paulista de M edicina-U N IFESP). Treze pacientes com LPA fizeram uso de AT RA. A síndrome foi diagnosticada em cinco pacientes (38\%) com idade média de 29 anos e que estavam,em média, no décimo dia do uso de ATRA. Os achados clínicos mais freqüentes foram insuficiência respiratória, infiltrado pulmonar à radiografia e febre. Este relato chama a atenção para a necessidade do diagnóstico precoce dessa síndrome, com a introdução de dexametasona ao primeiro sinal no intuito de êxito terapêutico.
\end{abstract}

Palavras-chave: leucemia promielocítica aguda; diagnóstico; quimioterapia; ácido transretinóico; síndrome AT RA.

\begin{abstract}
Acute promyelocytic leukemia (APL) is a subtype of acute myeloid leukemia (AML) responsible for $10 \%$ of all AM Ls. U sually, the treatment of APL consists of chemotherapy and all-trans retinoic acid (ATRA) administration. The major side effect of ATRA is the socalled "AT RA syndrome", which has an incidence of nearly $30 \%$. We studied the clinical presentation and the incidence of ATRA syndrome in patients with APL admitted to Hospital São Paulo-U N IFESP-EPM. Thirteen patients with APL received ATRA. ATRA syndrome was diagnosed in 5 patients (38\%) with a mean age of 29 years old, which were, in average, in the 10th day of the use of ATRA. The most frequent clinical findings were respiratory distress, pulmonary infiltrate on chest X-Ray and fever. This report calls attention to the need of early diagnosis of the syndrome with prompt introduction of dexamethasone at the first sign in order to obtain therapeutic success.
\end{abstract}

Key words: acute promyelocytic leukemia; diagnosis; chemotherapy; all-trans retinoic acid; ATRA syndrome.

${ }^{1}$ Residente em H ematologia. Universidade Federal de São Paulo, Escola Paulista de M edicina (U N IFESP-EPM ). Enviar correspondência para R.F.P. Avenida Dr. Altino Arantes 648 apto 11, Vila Clementino; 04042003 São Paulo, SP - Brasil.

E-mail: ronaldfeitosa@bol.com.br

2Pós-graduando em H ematologia. U niversidade Federal de São Paulo, Escola Paulista de M edicina (UN IFESP-EPM ), São Paulo, SP - Brasil. ${ }^{3} \mathrm{M} \mathrm{D}, \mathrm{PhD}$, Professor, Disciplina de $\mathrm{H}$ ematologia e H emoterapia, Universidade Federal de São Paulo, Escola Paulista de M edicina (U N IFESP-EPM ), São Paulo, SP - Brasil.

${ }^{4} \mathrm{M} \mathrm{D}, \mathrm{PhD}$, Professor, Disciplina de $\mathrm{H}$ ematologia e H emoterapia, Universidade Federal de São Paulo, Escola Paulista de M edicina (U N IFESP-EPM ), São Paulo, SP - Brasil. 


\section{INTRO DUÇÃO}

A leucemia promielocítica aguda (LPA) é um tipo específico de leucemia aguda caracterizada pelo predomínio de células com morfologia característica pela translocação $t(15,17)^{1}$ e por coagulopatia, que combina coagulação intravascular disseminada e fibrinólise. ${ }^{2} E$ designada M 3 pela classificação $F A B^{3,4}$ e LPA com $\mathrm{t}(15,17)$ ou rearranjo PM L/RARA pela O rganização Mundial de Saúde. ${ }^{5}$

A LPA é uma doença peculiar, responsável por cerca de $10 \%$ das leucemias mielóides agudas no adulto. Aproximadamente $95 \%$ dos casos apresentam a translocação balanceada entre os cromossomos 15 e 17 envolvendo 0 rearranjo dos genes PML e RARA. ${ }^{5-7} \mathrm{~A}$ adição do ATRA ao tratamento da LPA melhorou significativamente 0 nível de remissão completa.

0 mecanismo de ação da droga proposto é a indução à diferenciação dos precursores leucêmicos em células maduras. O ATRA exerce um efeito negativo no complexo PM L/RARA, quebrando a proteína quimérica além do complexo nuclear diacetil-histona. ${ }^{6}$ A té o início da década de 90, o tratamento da LPA era à base de antracíclico combinado com citarabina, mas altos índices de morte eram observados devido a distúrbios de sangramento. 8,9

Após a observação experimental de que o ATRA induzia diferenciação in vitro e in vivo, ${ }^{10,11} 0$ tratamento da LPA passou a ser realizado com a adição de AT RA, levando a um aumento no índice de remissão e redução no índice de recaída, conforme apresentado em estudos clínicos randomizados. ${ }^{12-14}$

No início da década de 90, o tratamento da LPA passou a ser realizado com a adição do ácido transretinóico (AT RA). 0 AT RA tem sido bem tolerado, exceto pelo desenvolvimento de uma síndrome denominada ATRA por Frankel et al ${ }^{15}$ em 1992, que consiste de febre, insuficiência respiratória e infiltrado pulmonar.

O objetivo do trabalho é avaliar a apresentação clínica, incidência e resposta ao tratamento da Síndrome ATRA nos pacientes admitidos no Hospital São Paulo (da Escola Paulista de M edicina-U N IFESP).

\section{MATERIAL E MÉTO DOS}

N o período de janeiro de 1991 a novembro de 2001, 13 pacientes com LPA receberam ATRA no $\mathrm{H}$ ospital São Paulo (da Escola Paulista de M edicina-U N IFESP).

O diagnóstico levou em conta a análise citológica de esfregaço de medula óssea, citoquímica, imunofenotipagem e cariótipo convencional por bandamento G. A partir do diagnóstico, o tratamento foi iniciado com daunorrubicina $\left(45 \mathrm{mg} / \mathrm{m}^{2}\right)$ via endovenosa em infusão de 3 horas, por três dias, citarabina $\left(100 \mathrm{mg} / \mathrm{m}^{2}\right)$ em infusão contínua via endovenosa por 24 horas, por sete dias e ATRA $\left(45 \mathrm{mg} / \mathrm{m}^{2}\right)$, diariamente, por via oral. 0 ATRA era mantido até 0 paciente atingir recuperação medular, isto é, plaquetas acima de 150.000/uL e segmentados acima de 1500/uL, o que significou o seu uso por aproximadamente três semanas. A Síndrome ATRA era diagnosticada na presença de pelo menos três dos seguintes critérios: febre, ganho de peso, insuficiência respiratória, infiltrado pulmonar, derrame pleural ou pericárdico, hipotensão ou insuficiência renal. Todos os pacientes apresentaram hemocultura e urinocultura negativas, um critério essencial para o diagnóstico da Síndrome ATRA. ${ }^{16}$

\section{RESULTADO S}

Através dos critérios mencionados, a Síndrome AT RA foi detectada em cinco pacientes, três mulheres e dois homens. A idade média foi de 29 anos (variando de 19 a 38 anos) e a leucometria média na apresentação da Síndrome foi de 3.500/uL (variando de 300 a 8.200 / uL). O s pacientes estavam, em média, no décimo dia do uso do ATRA. O s achados clínicos mais comumente encontrados foram insuficiência respiratória, infiltrado pulmonar na radiografia de tórax, febre, derrame pleural, insuficiência renal, derrame pericárdico e insuficiência cardíaca congestiva (Tabela 1). Remissão completa foi detectada em dois pacientes que desenvolveram a Síndrome AT RA. O s outros três casos não foram avaliados devido à morte precoce.

Tabela 1. Comparação das características e apresentação clínica de pacientes com síndrome ATRA.

\begin{tabular}{|l|c|c|c|c|c|c|c|c|}
\hline & Incidência & Febre & $\begin{array}{c}\text { Insuficiência } \\
\text { respiratória }\end{array}$ & $\begin{array}{c}\text { Insuficiência } \\
\text { renal }\end{array}$ & $\begin{array}{c}\text { Derrame } \\
\text { pleural }\end{array}$ & $\begin{array}{c}\text { Infiltrado } \\
\text { pulmonar }\end{array}$ & $\begin{array}{c}\text { Derrame } \\
\text { pericárdico }\end{array}$ & $\begin{array}{c}\text { Insuficiência } \\
\text { cardíaca }\end{array}$ \\
\hline $\begin{array}{l}\text { Frankel et al, } \\
1992\end{array}$ & $26 \%$ & $100 \%$ & $100 \%$ & $\sim 66 \%$ & $100 \%$ & $100 \%$ & $\sim 11 \%$ & $0 \%$ \\
\hline $\begin{array}{l}\text { Botton et al, } \\
1998\end{array}$ & $15 \%$ & $81 \%$ & $89 \%$ & $39 \%$ & $47 \%$ & $81 \%$ & $19 \%$ & $17 \%$ \\
\hline $\begin{array}{l}\text { Tallman et al, } \\
2000\end{array}$ & $26 \%$ & $81 \%$ & $84 \%$ & $11 \%$ & $36 \%$ & $52 \%$ & $36 \%$ & $11 \%$ \\
\hline $\begin{array}{l}\text { Pinheiro et al. } \\
2001\end{array}$ & $38 \%$ & $3 / 5$ & $5 / 5$ & $2 / 5$ & $3 / 5$ & $4 / 5$ & $3 / 5$ & $2 / 5$ \\
\hline
\end{tabular}




\section{DISCUSSÃO}

O uso do ATRA apresenta uma série de efeitos colaterais tais como pele seca, aumento de transaminases e triglicerídeos, eritema nodoso, cefaléia, pseudotumor cerebral, hipercoagulabilidade, basofilia, Síndrome de Sweet, vasculite necrotizante e até gangrena de Fournier. ${ }^{17}$ Porém, o principal efeito colateral dessa droga tem sido a SíndromeAT RA, uma entidade descrita em meados de 1990 que consiste de lesão pulmonar associada a outros sintomas sistêmicos. ${ }^{15}$

A incidência da Síndrome ATRA tem variado de $9 \%$ a $26 \% \cdot{ }^{17} \mathrm{~N}$ a sua descrição inicial, Frankel et al ${ }^{15}$ observaram incidência de $26 \%$ em 35 pacientes, enquanto $D$ e Botton et al ${ }^{16}$ descreveram em $15 \%$ de um total de 413 pacientes e Tallman et al ${ }^{18}$ - estudando 44 pacientes observaram uma incidência de $26 \%$. A incidência em nosso serviço foi superior às de Frankel et al, ${ }^{15} \mathrm{D}$ e Botton et al ${ }^{16}$ e Tallman et al. ${ }^{18}$ Acreditamos que isso se deva ao pequeno número de casos em nosso relato.

A apresentação clínica mais freqüente da Síndrome AT RA foi a insuficiência respiratória com radiografia de tórax com padrão retículo-nodular bilateralmente. 0 utros sinais e sintomas encontrados foram, principalmente, febre, derrame pleural, pericárdico e sinais de insuficiência cardíaca. $\mathrm{N}$ a descrição inicial da Síndrome ATRA também foi predominante 0 envolvimento do sistema pulmonar, ${ }^{15}$ apesar da descrição de outros sinais como ganho de peso, derrame pleural, derrame pericárdico e hipotensão. Assim, fica bastante evidente que a apresentação clínica mais comum dessa síndrome é a insuficiência respiratória e a febre, sendo o pulmão o órgão mais comumente lesado e injúria pulmonar o maior problema no desenvolvimento da síndrome. Com o passar dos anos, foram acrescentados outros aspectos à síndrome como choque cardiogênico, tamponamento cardíaco, ${ }^{19}$ hemorragia alveolar difusa com capilarite pulmonar, ${ }^{20}$ pneumotórax ${ }^{21}$ e íleo paralítico. $^{22}$

A patogênese da Síndrome ATRA ainda não foi totalmente elucidada. Achados de autópsia têm apresentado edema, hemorragia, exsudato fibrinoso e infiltração de leucócitos, determinando uma destruição da microvasculatura pulmonar. ${ }^{23} 0$ resultado final desse processo é uma lesão endotelial com exsudato fibrinoso e infiltração de neutrófilos, levando à insuficiência respiratória. Acredita-se que no mecanismo de injúria tissular haja participação de mediadores inflamatórios como a catepsina $G$ secretada pelos granulócitos, promovendo vasodilatação e aumento da permeabilidade capilar. ${ }^{18}$
Em um paciente que esteja em uso de ATRA e apresente insuficiência respiratória ou febre, deve-se introduzir corticoterapia para tratamento da síndrome, pois essa é a única maneira de evitar a evolução grave. Porém, deve-se lembrar que essa apresentação clínica não pode ser diferenciada de uma infecção pulmonar por um bacilo gram-negativo ou por "Pneumocistis carinii", sendo importante ampla cobertura antibiótica contra bactéria gram-negativo.

0 tratamento da síndrome ATRA tem sido à base de corticoterapia desde sua descrição inicial. ${ }^{15}$ Frankel et al ${ }^{15}$ trataram pacientes com dexametasona $10 \mathrm{mg}$ duas vezes ao dia por um mínimo de três dias e conseguiram reverter e controlar o quadro clínico dentro de poucas horas. D esde então, essa dose de dexametasona vem sendo preconizada em uma série de trabalhos que confirmam o benefício desse corticóide, principalmente se introduzido ao primeiro sinal da síndrome. ${ }^{16,18}$ Essa conduta foi realizada em dois pacientes do nosso serviço com boa resposta.

0 aparecimento da síndrome em nosso serviço foi em torno do décimo dia do uso do AT RA. 0 utros relatos apresentam a síndrome em torno do décimo primeiro, décimo segundo e décimo quarto dia, com um intervalo do segundo ao vigésimo primeiro dia. ${ }^{15,16,18}$

$\mathrm{N}$ a descrição inicial da síndrome, Frankel et al ${ }^{15}$ relataram que o início da síndromeAT RA fora precedido por um aumento na leucometria do sangue periférico (LSP) em seis dos nove casos. Tem sido sugerido que pacientes com LSP que supere 5.000/uL no dia 01, 6.000/uL no dia 05, 10.000/uL no dia 10 ou 15.000/ uL no dia 15 têm um risco aumentado da síndrome AT RA. ${ }^{14}$ Tallman et $a^{18}$ criaram um modelo variante para predizer o valor da LSP no dia 01 de uso da droga para 0 desenvolvimento da síndrome AT RA nos dias 05,10 e 15 de uso da droga. Esse modelo não apresentou nenhuma diferença entre os que desenvolviam ou não a síndrome. $\mathrm{N}$ ão houve relação entre a LSP e 0 desenvolvimento da síndrome AT RA no presente relato.

$\mathrm{N}$ a realidade, a letalidade da síndrome AT RA no nosso estudo foi alta devido principalmente ao atraso na identificação desta nos três primeiros pacientes em meados dos anos 90 . N esse período, os trabalhos iniciavam a descrição dessa complicação e pouco se sabia sobre a síndrome ATRA. Um desses pacientes necessitou de hemodiálise e cinco precisaram de suporte ventilatório, exatamente as mesmas complicações apresentadas em outros trabalhos. ${ }^{15,16,18}$ D ois pacientes tiveram melhora em 72 horas após o uso dexametasona esuporteventilatório. A letalidadeobservada por Frankel et al. ${ }^{15}$ foi de três mortes num universo de 35 doentes. $\mathrm{N}$ os 64 pacientes relatados por $\mathrm{D}$ e Botton et al, ${ }^{16}$ nove 
mortes foram diretamente atribuídas à Síndrome AT RA, 13 pacientes necessitaram de ventilação mecânica e somente dois fizeram uso de tratamento dialítico.

Esse relato chama atenção para a necessidade do diagnóstico precoce da síndrome ATRA, devendo ser imediatamente introduzida a dexametasona para se al cançar o sucesso terapêutico.

\section{CONCLUSÃO}

A Síndrome ATRA é hoje o principal efeito colateral do uso do ATRA no tratamento da LPA. Essa síndrome consiste basicamente em febre, ganho de peso, insuficiência respiratória, infiltrado pulmonar, derrame pleural ou pericárdico, hipotensão ou insuficiência renal. O s principais achados clínicos em nossos pacientes foram insuficiência respiratória, infiltrado pulmonar e febre (Tabela 1). 0 tratamento éà base de dexametasona $10 \mathrm{mg}$ duas vezes ao dia, por no mínimo três dias. N os pacientes do nosso serviço em que a síndrome foi reconhecida, a utilização de dexametasona foi fundamental para a boa evolução dos casos. Consideramos que corticóide deve ser introduzido ao primeiro sinal da síndrome.

\section{REFERÊNCIA BIBLIO GRÁFICA}

1. Larson RA, Kondo K, Vardiman JW, Butler ARE, Golomb H M , Rowley JD . Evidence for a $(15 ; 17)$ translocation in every pacient with acute promyelocytic leukemia. Am J M ed 1984;76:827.

2. Tallman M S, Kwaan HC. Reassessing thehemostatic disorder associated with acutepromyelocytic leukemia. Blood 1992;79:543.

3. Bennett JM , C atowsky D , D aniel M T, Flandrin G, G alton $D A, G$ ralnick $H R$, et al. Proposalsfor the classification of the acuteleukemias. BrJ H aematol 1976;33(4):451-8.

4. Bennet $J M, C$ atowsky D, D aniel M T, Flandrin $G, G$ alton $D A, G$ ralnick $H R$, et al. A variant form of hypergranular promyelocytic leukemia (M 3). Ann Intern M ed 1980;92:28.

5. H arris $N \mathrm{~L}$, Jaffe $E S, D$ iebold J, Flandrin $G$, M ullerH ermelink HK, Vardiman J, et al. World $\mathrm{H}$ ealth $\mathrm{O}$ rganization, classification of neoplastic disease of thehematopoietic and lymphoid tissues: report of the Clinical Advisory Committee meeting-Airlie $\mathrm{H}$ ouse, Virginia, N ovember 1997. J Clin O ncol 1999;17(12):3835-49.

6. Willman CL. M olecular evaluation of acutemyeloid leukemias. Semin H ematol 1999;36(4):390-400.

7. ChauffailleM LLF, Figueiredo M S, Beltrani R, AntunesSV, Yamamoto $M$, K erbauy J. Acutepromyelocytic leukemia: the study of $(15 ; 17)$ translocation by FISH , RT-PCR, and cytogenetic techniques. BrazJ M ed Biol Res 2001;34:735-43.
8. Cunningham I, GeeT, Reich L. Acute promyelocytic leukemia: treatment results during a decade at the M emorial H ospital. Blood 1989;73:1116.

9. Kantarjian $\mathrm{H}$, Keating $M$, WaltersR. Acute promyelocytic leukemia M D Anderson H ospital experience. Am J M ed 1986;80:789.

10. ChomienneC, Ballerini P, Balitrand N, D aniel M T, Fenaux $\mathrm{P}, \mathrm{C}$ astaigneS, et al. All trans retinoic acid in promyelocytic leukemias. In vitro studies structure function relationship. Blood 1990;76:1710.

11. CastaigneS, Chomienne C, D aniel M T, Berger R, Fenaux $P, D$ egos L. All transretinoic acid as a differentiating therapy for acute promyelocytic leukemia: clinical results. Blood 1990;76:1704.

12. Fenaux P, Le D eley M C, Castaigne S. Effect of all transretinoic acid in newly diagnosed acute promyelocytic leukemia: results of a multicenter randomized trial. Blood 1993;82:3241.

13. Fenaux P, Wattel E, Archimbaud E, Sanz M , H ecquet B, Guerci $A$, et al. Prolonged follow up confirms that all transretinoic acid (AT RA) followed by chemotherapy reduces the risk of relapse in newly diagnosed acute promyelocytic leukemia (APL). Blood 1994;84:666.

14. Tallman M S, Andersen JW, Schiffer CA, Appelbaum FR, Feusner $\mathrm{H}$, $\mathrm{O}$ gden $\mathrm{A}$, et al. All trans retinoic acid in acute promyelocytic leukemia. $N$ Eng J Med 1997;337(15):1021-8.

15. Frankel SR, Eardley A, Lauwers G, Weiss M , Warrell R. The retinoic acid syndromein acutepromyelocytic leukemia. Ann Intern M ed 1992;117:292.

16. D eBotton S, D ombret H, Sanz M , M iguel JS, C aillot D, Zittoun $R$, et al. Incidence, clinical features, and outcome of all trans-retinoic acid syndrome in 413 cases of newly diagnosed acute promyelocytic leukemia. Blood 1998;92(8):2712-8.

17. Fenaux $P$, C homienne C, D egosL. All-trans retinoic acid and chemoterapy in the treatment of acutepromyelocytic leukemia. Semin H ematol 2001;38(1):13-25.

18. Tallman M S, Andersen JW, Schiffer CA, Appelbaum FR, Feusner JH , O gden A, et al. Clinical description of 44 patients with acute promyelocytic leukemia who developed theretinoic acid syndrome. Blood 2000;95(1):90-5.

19. Larrea L, de la Rubia J, Jimenez C, M artin G, Sanz M A. Cardiac tamponadeand cardiogenic shock as a manifestation of all transretnoic acid syndrome: an association not previously reported. H aematologica 1997;82(4):463.

20. N icollsM R, TeradaLS, Tuder RM , PrindivilleSA, Schwarz M I. D iffuse alveolar hemorrhagewith underlying pulmonary capillaritis in theretinoic acid syndrome. AmJ Respir C rit Care M ed 1998;158(4):1302-5.

21. D avis BA, Cervi P, Amin Z, M oshi G, Shaw P, Porter J. Retinoic acid syndrome: pulmonary computed tomography (CT) findings. Leuk Lymphoma 1996;23(1/2):113. 
22. KanisawaY, Kawanishi $\mathrm{N}, \mathrm{H}$ isai $\mathrm{H}$, Araya H , AkiyamaT, Takahari $D$. Paralytic ileus during treatment of acute promyelocytic leukemia with all transretinoic acid. Rinsho Ketsueki 2000;41(8):687.
23.Feng D, N agy JA, Pyne K, D vorak H F, D vorak AM . $\mathrm{N}$ eutrophils emigratefrom venules by a transendothelial cell pathway in response to FMLP. J Exp Med 1998;189:903. 